\title{
Development of simple and accurate voltammetric method based on Surfactant Modified Graphene for the Determination of Rutin
}

\author{
Nagaraja Sreeharsha ${ }^{1,2, *}$, Mallikarjun Telsang, ${ }^{3, *}$, Girish Meravanige Basavarajappa ${ }^{4}$, \\ Swati Pund ${ }^{5}$ \\ ${ }^{1}$ Department of Pharmaceutical Sciences, College of Clinical Pharmacy, King Faisal University, Al- \\ Ahsa 31982, Saudi Arabia; \\ ${ }^{2}$ Department of Pharmaceutics, VidyaSiri College of Pharmacy, Off Sarjapura Road, Bangalore \\ 560035, Karnataka, India \\ ${ }^{3}$ Department of Surgery, College of Medicine, King Faisal University, Al-Ahsa 31982, Saudi Arabia; \\ ${ }^{4}$ Department of Biomedical Sciences, College of Medicine, King Faisal University, Al-Ahsa 31982, \\ Saudi Arabia; \\ ${ }^{5}$ Nanomedicine Laboratory, Department of Biosciences and Bioengineering, Indian Institute of \\ Technology-Bombay, Mumbai 400076, India; \\ *E-mail: sharsha@kfu.edu.sa, mvtelsang@kfu.edu.sa
}

Received: 11 November 2021 / Accepted: 17 December 2021 / Published: 5 January 2022

This examination relates to the electrochemical analysis of Rutin (RT) at anionic surfactant Sodium dodecyl sulfate chemically modified graphene paste electrode (SDSMGPE) and its electrocatalytic oxidation or reduction were analyzed using the cyclic voltammetry (CV). The fabricated electrode showed an impressive electrocatalytic movement for RT with a decent electrochemical performance, low over potential and high conductivity. It was found that under ideal conditions (pH7.0) in CV, the oxidation potential of RT decrease to $291 \mathrm{mV}$ at the SDSMGPE as compared to a bare graphene paste electrode (BGPE). Calibration plot showed two direct unique reaches of $2.0-10 \mu \mathrm{M}$ and 10-60 $\mu \mathrm{M}$ with a calculated detection limit (LOD) and calculated limit of quantification (LOQ) are found to be $1.17 \times 10^{-}$ ${ }^{8} \mathrm{M}$ and $3.91 \times 10^{-8} \mathrm{M}$, respectively. Simultaneous investigation was completed with paracetamol (PC), effectively shows two obvious sharp tops with improved current as compared to the BGPE. The SDSMGPE has excellent reproducibility and long term stability with fine recovery in real sample of RT. Field emission scanning electron microscopy (FESEM) examination was performed to check the surface morphology of the BGPE and SDSMGPE and EIS was utilized to explore the electrode and solution interface properties.

Keywords: Rutin; Paracetamol; Graphene sensor; Electroanalysis. 
(C) 2022 The Authors. Published by ESG (www.electrochemsci.org). This article is an open access article distributed under the terms and conditions of the Creative Commons Attribution license (http://creativecommons.org/licenses/by/4.0/). 